\title{
Publisher Correction: Glycine max NNL1 restricts symbiotic compatibility with widely distributed bradyrhizobia via root hair infection
}

Bao Zhang, Mengdi Wang, Yifang Sun, Peng Zhao, Chang Liu, Ke Qing, Xiaotong Hu, Zhedong Zhong, Jialong Cheng, Haijiao Wang, Yaqi Peng, Jiajia Shi, Lili Zhuang, Si Du, Miao He, Hui Wu, Min Liu, Shengcai Chen, Hong Wang, Xu Chen, Wei Fan, Kewei Tian, Yin Wang, Qiang Chen, Shixiang Wang, Faming Dong, Chunyan Yang, Mengchen Zhang, Qijian Song, Youguo Li and Xuelu Wang (iD

Correction to: Nature Plants https://doi.org/10.1038/s41477-020-00832-7, published online 15 January 2021.

In the version of this Article originally published, Youguo Li's affiliation with the Center of Integrative Biology was mistakenly omitted; their affiliations should have been: Center of Integrative Biology, College of Life Science and Technology, Huazhong Agricultural University and State Key Laboratory of Agricultural Microbiology, College of Life Science and Technology, Huazhong Agricultural University. This has now been corrected.

Published online: 8 February 2021

https://doi.org/10.1038/s41477-021-00872-7

(c) The Author(s), under exclusive licence to Springer Nature Limited 2021 